\title{
EDUCACIÓN FINANCIERA Y SU EFECTO EN EL INGRESO EN MÉXICO
}

\begin{abstract}
Alejandro Mungaray, ${ }^{a}$ Nidia Gonzalez ${ }^{b}$ y Germán Osorio ${ }^{b}$
Fecha de recepción: 17 de junio de 2020. Fecha de aceptación: 9 de diciembre de 2020.

https://doi.org/10.22201/iiec.20078951e.2021.205.69709

Resumen. La educación financiera es un factor importante para incrementar los niveles de bienestar en países con alto grado de desigualdad, pues permite a los ciudadanos mejorar su participación en las actividades económicas a través de decisiones financieras. En el presente trabajo se construye un índice de educación financiera para México. El modelo econométrico demuestra que el aumento en el índice de educación financiera es relevante para aumentar el ingreso mensual promedio de los mexicanos. Los resultados sugieren que el rezago en educación financiera de la población mexicana radica en la escaza inclusión social debido a la desigualdad y los altos niveles de endeudamiento, ya que esto limita que los esfuerzos públicos o privados realizados tengan éxito.
\end{abstract}

Palabras clave: endeudamiento; educación financiera; bienestar; desigualdad; inclusión social.

Clasificación JEL: D63; P43; P46.

\section{FINANCIAL EDUCATION AND ITS EFFECT ON INCOME IN MEXICO}

\begin{abstract}
Financial education has an important role to play in increasing welfare levels in countries with a high degree of inequality, as it enables citizens to make financial decisions that increase their share of economic activities. This article constructs a financial education index for Mexico. The econometric model shows that an increase in the financial education index influences the average monthly income of Mexicans. The findings suggest that the lag in the Mexican population's financial education results from the lack of social inclusion caused by inequality and high levels of indebtedness, a reality that limits the success of both public or private interventions.
\end{abstract}

Key Words: indebtedness; financial education; welfare; inequality; social inclusion.

\footnotetext{
${ }^{a}$ Universidad de Sonora, Departamento de Economía; ${ }^{\mathrm{b}}$ Universidad Autónoma de Baja California, Facultad de Economía y Relaciones Internacionales. Correos electrónicos: mungaray@uabc.edu.mx; nidia.gonzalez@uabc.edu.mx y gosorio@uabc.edu.mx, respectivamente.
} 


\section{INTRODUCCIÓN}

La educación financiera es un proceso por el cual los individuos adquieren capacidades y habilidades en mercados de dinero permitiendo, entre otras cosas, mejorar la comprensión de la economía, el acceso a productos bancarios y a oportunidades de inversión.

En México, según la última Encuesta Nacional de Inclusión Financiera de la Comisión Nacional Bancaria y de Valores (CNBv-enif, 2015), 52.9\% de la población no tiene relación alguna con instituciones financieras; $47.5 \%$ sólo dispone de ahorros informales; mientras que $44.9 \%$ no considera tener ingresos suficientes para adquirir productos financieros, y $21.5 \%$ nunca ahorra. Esta información revela la importancia de acciones y estrategias, públicas y privadas, que promuevan mayores niveles de educación financiera.

En tanto, la evidencia empírica afirma que, para incrementar la educación financiera en los países, es necesaria la colaboración tanto de entes públicos como privados que permita la participación de empresas privadas en el fomento de talleres y programas de acceso público a cualquier segmento de la población. De hecho, las propias instituciones financieras deben lograr encadenamientos de futuros usuarios hacia productos financieros y su correcta aplicación. Para lograr mayores niveles en educación financiera, las personas deben conocer los efectos que tienen sus decisiones en su bienestar económico y social (Diez, 2009).

A nivel internacional, la Organización para la Cooperación y el Desarrollo Económicos (Organización para la Cooperación y el Desarrollo Económicos [OCDE], 2017) calcula un Indicador de Educación Financiera (IEF) a través de una suma de tres componentes: conocimientos, actitudes y comportamientos financieros. ${ }^{1}$ En este sentido, los países que integran el G20 presentan un índice, en promedio, de $60.47 \%$. Francia se encuentra en primer lugar con $70.95 \%$, seguido por Noruega y Canadá con $69.52 \%$, China con $67.14 \%$ y Corea con $66.19 \%$. Por su parte, México tiene un índice de $57.62 \%$, ubicándose en la posición 12, por debajo de países como Indonesia con $63.80 \%$ (OCDE, 2017).

1 Por conocimiento financiero se entiende al conocimiento básico de inflación, diversificación, intereses sobre un préstamo, cálculos de interés y retorno; por actitudes financieras se refiere a priorizar las necesidades y ahorro futuro; y por comportamiento financiero a pensar antes de realizar una compra, pagar las cuentas a tiempo y presupuestar, ahorrar y pedir préstamos (Atkinson y Messy, 2012). 
Una barrera para lograr aumento en los niveles de educación financiera son, paradójicamente, la constante modernización del sector financiero donde las personas con bajos conocimientos y educación se enfrentan, por lo general, a procesos que pueden desincentivar el consumo de los productos disponibles en el mercado. Así, entre más sofisticación, mayores deben ser las capacidades de la población para aprovecharlas y reducir la desigualdad, a través de la educación financiera. Aprovechar o no las oportunidades de inversión financiera refleja cambios en la desigualdad y en el desarrollo financiero (Jappelli, 2010; Lo Prete, 2017). De seguir con la misma tendencia, la brecha de desigualdad continuará expandiéndose y las capacidades de un sistema financiero óptimo en México, donde un agente económico pueda acceder al mercado con los conocimientos suficientes, seguirán siendo limitadas para mejorar el nivel de bienestar y la inclusión social de sus habitantes.

Se ha comprobado que la desigualdad social es menor en los países donde la educación financiera es más alta (Lo Prete, 2013), y que un mayor conocimiento económico amplía la conciencia y cambia las actitudes, modificando el comportamiento social en general (Carpena et al., 2011). La educación financiera es relevante para fomentar medidas de inclusión en todos los estratos sociales. Mediante la aplicación correcta de los instrumentos financieros disponibles es posible impactar positivamente en el bienestar de los hogares, especialmente si se refleja en la capacidad de los individuos para formular estrategias que fortalezcan sus conocimientos/habilidades en la toma de decisiones para uso y funcionamiento de sus recursos financieros y no financieros (Widdowson y Hailwood, 2007; Lusardi et al., 2017).

Diversos estudios coinciden en que el crecimiento económico en México es limitado y no logra atender las necesidades de la población. Aparicio (2010) argumenta que desde hace tres generaciones la economía mexicana creció, en promedio, sólo 2\% cada año (De la Cruz y Veintimilla, 2013; Sánchez y Moreno, 2016; BANXICO, 2018). La reforma financiera logró aumentar la oferta de crédito a la población, pero sin garantía de crecimiento sostenido, debido a "la precariedad en educación financiera de la población" (De la Cruz y Alcántara, 2011; De la Cruz y Veintimilla, 2013).

De ahí que las tarjetas de crédito constituyan uno de los principales canales de crédito al consumo, y que en 2019 representaran 38.6\% de la cartera total del crédito al consumo. Mientras que $61.3 \%$ de los consumidores suele no aprovechar promociones y prefieren realizar sus compras pagando intereses sin plazos preferenciales, $48.78 \%$ suele pagar intereses a las instituciones (BANXICo, 2019). Lo que significa que la oferta de créditos aumentó ligeramente en 
el país, pero que también que los consumidores no cuentan con las habilidades necesarias para hacer uso eficiente de los productos financieros.

Ahora bien, respecto al término de inclusión social, se refiere al proceso de cambio que permite la "participación" progresiva a nivel social, económico y político de la población (Araoz-Fraser, 2010). Por otro lado, el Banco Mundial (2014) la define como un proceso por el cual se puede mejorar la habilidad y la oportunidad de las personas en situación de desventaja (desigualdad o pobreza), para que puedan participar en diferentes ámbitos de la sociedad. En este trabajo inclusión social se asocia a la "participación plena" de cualquier individuo, al acceder a la educación financiera y al sistema financiero, ya que permite resaltar la participación de cualquier consumidor sin ningún tipo de discriminación al núcleo social al que pertenece.

El objeto de esta investigación es medir el impacto que la educación financiera genera en los ingresos de los mexicanos, en personas entre 18 a 70 ańos, capaces de tomar decisiones financieras. Se asume la hipótesis de que un(a) jefe(a) de familia con mayor nivel de educación financiera obtiene aumentos significativos en sus ingresos.

Esta hipótesis se contrasta siguiendo la metodología de la OCDE, para construir un IEF con información de la CNBV-INEgi-enif, 2018. Para ello, se estructuran variables derivadas de reactivos relacionados con tres factores: actitudes, conocimiento y comportamiento financieros. Posteriormente, se desarrolla un modelo econométrico de sección cruzada donde se relaciona el IEF y diferentes niveles de ingreso, con el fin de conocer la posible causalidad y magnitud de relación, a través del uso de variables de control que reflejen el perfil sociodemográfico de los individuos.

El trabajo se estructura en cinco apartados. En el primero se encuentra la introducción del trabajo, en el segundo se esboza la revisión de literatura, en el tercer apartado se presenta la metodología empleada; en el cuarto se discuten los resultados, para finalmente llegar a las conclusiones y posibles líneas futuras de investigación.

\section{IMPORTANCIA DE LA EDUCACIÓN FINANCIERA}

La investigación tradicional en materia de educación financiera se enfoca, sobre todo, en estudios sobre los hábitos de consumo, ahorro, planeación para el retiro y administración financiera de los hogares que esbozaban empíricamente el comportamiento financiero de los individuos. Es en 2005 cuando organismos internacionales, liderados por la OCDE, externan su preocupación 
por incrementar los niveles de educación financiera en la población, a la luz del escaso conocimiento financiero que tienen los consumidores para mejorar su bienestar económico.

La educación financiera se define como el proceso por el cual los consumidores o inversionistas financieros mejoran su comprensión de los productos financieros, conceptos y riesgos, con el fin de desarrollar habilidades y confianza, para tomar decisiones informadas sobre a dónde ir para obtener ayuda y ejercer cualquier acción eficaz para mejorar su bienestar económico (OCDE-CVM, 2005). Estudios para países desarrollados -como Estados Unidos- demuestran que existen niveles altos de analfabetismo financiero en algunos sectores de la población, sobre todo, en los agentes económicos de la tercera edad, de quienes se espera tomen decisiones financieras para asegurar su patrimonio hacia el final de su ciclo de vida (Lusardi, 2008). De acuerdo con Cartagena (2008), al promover que los usuarios del sistema financiero (tanto activos como potenciales) puedan tomar decisiones financieras mejor informados, permite resolver problemas de fraudes y mejorar el bienestar de la población. Este potencial de la educación financiera la convierte en una prioridad para instituciones públicas, organizaciones internacionales, instituciones multilaterales y foros internacionales (García et al., 2013).

En un estudio realizado por García-Suaza et al. (2009) reportan que a mayor edad un individuo puede invertir en un proyecto financiero como la educación consistente con la teoría del capital humano; otros estudios como los de Lusardi y Mitchell (2014) y Lusardi et al. (2017) modelan el conocimiento financiero como un tipo de inversión en capital humano, pues representa la capacidad de las personas para procesar información económica y tomar decisiones informadas sobre planificación financiera, acumulación de riqueza, deuda y pensiones. Este argumento se demuestra en un estudio sociodemográfico en donde se concluye que la administración financiera tiene un impacto sobre el bienestar financiero, mejorando sus ingresos hasta $86.2 \%$ para ciertas actividades económicas (Mejía, 2016). Otros estudios muestran que, sin el conocimiento e incentivos adecuados, no hay un uso óptimo o responsable de los servicios financieros (Lusardi y Mitchell, 2014; Villagómez y González, 2014; Ceballos, 2018).

En consecuencia, se insiste en colocar la educación financiera con valor curricular en el sector educativo, como un componente fundamental para una ciudadanía que a largo plazo cuente con habilidades financieras para tomar mejores decisiones y obtener mayores beneficios (Huchín y Simón, 2011; Córdova-Rangel et al., 2016; Duque et al., 2016; Denegri et al., 2018). No obstante, este mecanismo presenta retos importantes, pues también se ha de- 
mostrado que, a pesar de que en los programas educativos se aplican conocimientos financieros con valor curricular, las estadísticas no reflejan resultados positivos (Villagómez e Hidalgo, 2017).

Algunas investigaciones atribuyen la falta de educación financiera a factores relacionados con la oferta y la demanda de servicios financieros. Desde el punto de vista de la oferta, la educación financiera permite a los hogares tomar decisiones sobre cómo invertir su riqueza y qué nivel de endeudamiento pueden tener sin comprometer su estabilidad económica, factores clave para provocar cambios en las decisiones financieras (Lusardi, 2012). Y desde el punto de vista de la demanda, los bajos niveles de ingreso y la carencia de conocimientos reducen el ahorro e impiden que los clientes potenciales obtengan beneficios de estos servicios incentivando la falta de inclusión financiera (Raccanello y Herrera, 2014).

Jappelli (2010) descubre en su estudio que un puntaje de altas capacidades matemáticas se correlaciona positivamente con la educación financiera y, a su vez, afectan el desarrollo financiero por medio de mayor inversión. En esa dirección, en otro estudio se reporta que la desigualdad disminuye en los países donde la educación financiera es más alta entre la población (Lo Prete, 2013). Otras líneas de investigación reflejan una brecha de género en la educación financiera, ya que las mujeres suelen contar con bajos niveles de educación financiera, son más impacientes, no comprenden su rol en la economía debido a diversos patrones culturales e incluso es posible la discriminación en el sector financiero (Lusardi y Mitchell, 2014; Arcos-Medina et al., 2016; DomínguezGijón et al., 2018; Federici, 2018; Larraz et al., 2019).

La educación financiera permite el incremento de la inclusión financiera, bajo una regulación apropiada, esquemas de protección al consumidor y conocimiento técnico para mejorar las capacidades financieras de todos los segmentos de la población (CNBV, 2012). Esto permite incrementar el bienestar de la población, al desplazar los flujos de ingreso y consumo en el tiempo por medio del ahorro y el crédito, así como la acumulación de activos y la creación de un fondo para la vejez (Raccanello y Herrera, 2014).

Carpena et al. (2011) concluyen que un mayor conocimiento financiero genera cambios positivos en las decisiones financieras. En consecuencia, cuanto mayor es el conocimiento financiero, más equitativa es la distribución de la renta, por lo que promover la educación financiera es un mecanismo adicional a las políticas redistributivas para mejorar la equidad económica (Oliver et al., 2017). Atkinson y Messy (2012) realizan un estudio para conocer el comportamiento dentro de los países pertenecientes al G20, y en el que Alemania, Malasia y Perú registran niveles de educación financiera altos y comporta- 
mientos positivos de la población, ya que analizan antes de realizar compras y administran a futuro.

Los beneficios de la educación financiera pueden extenderse a la economía en general, promoviendo las competencias necesarias para tomar decisiones informadas y apropiadas, así como proporcionar herramientas para que las personas tengan la capacidad de defender sus derechos como consumidores financieros (García et al., 2013). Así, la educación financiera genera capacidades en el ser humano para administrar, desarrollar y aprovechar conceptos y productos financieros, a la hora de elegir la forma de acercarse al recurso financiero, sea como emprendedores o consumidores (Sauza et al., 2018; Martínez, 2017; Céspedes, 2017).

\section{Metodología}

El modelo empleado en esta investigación implica la construcción de un IEF para México, siguiendo la metodología de Atkinson y Messy (2012), para la OCDE. Ésta considera tres factores: conocimiento, actitudes y comportamiento financieros. La información se recaba por medio de una encuesta aplicada a países pertenecientes al G20. Para el caso de México se utiliza la información de la CNBV-INEGI-ENIF 2018, seleccionando las preguntas con factores de interés de la OCDE, con una muestra de 12446 mexicanos.

El IEF se obtiene haciendo un recuento del número de respuestas correctas a las preguntas asociadas a los tres factores de interés; posteriormente se obtiene la suma de los tres puntajes para conseguir el valor final del índice (véase tabla 1). Con el fin de normalizar los datos y obtener la variable serial para fines del análisis, se multiplica por cien y se divide entre 21 consistente con las respuestas totales aplicadas en la encuesta de la OCDE.

El IEF se clasifica en bajo, medio y alto; además se obtienen cuantiles de ingreso. Para conocer el nivel de endeudamiento de los mexicanos, se recolectó información de la ENIF para créditos formales, considerando el número de tarjetas de crédito con los que cuentan los encuestados, el monto del pago que realizan y si tienen acceso a créditos adicionales. La codificación de las respuestas se realizó de acuerdo con los instrumentos aplicados por Rivera y Bernal (2018), para calcular qué tan endeudados están los mexicanos en 2018 (véase tabla 2).

Debido al tamaño de la muestra, se consideraron tres intervalos para distribuir la población en nivel bajo, medio y alto de endeudamiento (véase tabla 3), con valores de los intervalos que fluctúan de 0-2 (nivel bajo), 3-5 (nivel medio) e iguales o mayores a 6 (nivel alto). 


\section{Alejandro Mungaray, Nidia Gonzalez y Germán Osorio}

\section{Tabla 1. Índice de educación financiera}

\begin{tabular}{|c|c|c|c|}
\hline Factores & Tema & Número de pregunta & Valoración \\
\hline \multirow{7}{*}{$\begin{array}{l}\text { Conocimiento } \\
\text { financiero }\end{array}$} & Tiempo valor del dinero & 12.4 & \multirow{7}{*}{$\begin{array}{l}1 \text { para respuestas } \\
\text { correctas. } 0 \text { en } \\
\text { todos los demás } \\
\text { casos }^{\star \star}\end{array}$} \\
\hline & Intereses pagados sobre un préstamo & 12.1 & \\
\hline & Interés más principal/ Interés simple con cálculo & 12.2 & \\
\hline & Interés compuesto & 12.3 & \\
\hline & Riesgo y retorno & 4.9.2 & \\
\hline & Definición de inflación & 4.9.1 & \\
\hline & Diversificación & 4.9.1 & \\
\hline \multirow{7}{*}{$\begin{array}{l}\text { Comportamiento } \\
\text { financiero }\end{array}$} & Tiene un presupuesto familiar & 4.1 & \multirow{7}{*}{$\begin{array}{l}1 \text { para respuestas } \\
\text { correctas. } 0 \text { en } \\
\text { todos los demás } \\
\text { casos }^{\star \star}\end{array}$} \\
\hline & Ahorro activo & 5.1 & \\
\hline & Compra considerada & 4.8 .1 & \\
\hline & Pago puntual de facturas & 4.8.2 & \\
\hline & Establecimiento de objetivos financieros a largo plazo & 4.8 .4 & \\
\hline & Elegir productos* & $\begin{array}{l}5.21 ; 5.22 ; 6.16 \\
6.17 ; 8.13 ; 8.14\end{array}$ & \\
\hline & Préstamo para llegar a fin de mes* & 4.6 & \\
\hline \multirow{2}{*}{$\begin{array}{l}\text { Actitudes } \\
\text { financieras }\end{array}$} & Tiendo a vivir por hoy y dejar que el mañana se cuide solo* & 9.9 & $\mathrm{l}=\mathrm{Si} ; 0=\mathrm{N}_{0}$ \\
\hline & $\begin{array}{l}\text { Encuentro más satisfactorio gastar dinero que ahorrar para } \\
\text { el largo plazo }\end{array}$ & 4.8 .3 & $\mathrm{l}=\mathrm{Si} ; 2=\mathrm{N}_{0}$ \\
\hline
\end{tabular}

Notas: los temas, preguntas, respuestas y puntuación son definidos en Atkinson y Messy (2012); * el puntaje se basa en una variable derivada de información que considera preguntas relacionadas con el tema; ${ }^{\star \star}$ respuestas correctas difieren según la pregunta, para entender más sobre la valoración revisar (OCDE, 2017).

Fuente: elaboración propia con información de la CNBV-INEGI-ENIF, 2018. Disponible en https://www.inegi.org.mx/ programas/enif/2018/\#Datos_abiertos 


\section{Tabla 2. Nivel de endeudamiento}

\begin{tabular}{|c|c|c|}
\hline Preguntas & Opciones de respuesta & Valoración \\
\hline \multirow[t]{3}{*}{ ¿Cuántas tarietas de crédito posee? } & 1 & 0 \\
\hline & 2 & 1 \\
\hline & Más de 2 & 2 \\
\hline \multirow{3}{*}{$\begin{array}{l}\text { Durante el 2017, ¿ ise ha atrasado en } \\
\text { alguna mensualidad? }\end{array}$} & Nunca & 0 \\
\hline & Una vez & 1 \\
\hline & Más de una vez & 2 \\
\hline \multirow{2}{*}{$\begin{array}{l}\text { ¿Posee algún tipo de crédito o deuda } \\
\text { financiera distinto a la tarieta de crédito? }\end{array}$} & Sí & 1 \\
\hline & No & 0 \\
\hline \multirow{5}{*}{$\begin{array}{l}\text { ¿El pago que realiza a su tarjeta } \\
\text { generalmente cubre? }\end{array}$} & Requerido para no generar intereses 0 el total del adeudo & 0 \\
\hline & Más del pago mínimo & 1 \\
\hline & Pago mínimo & 2 \\
\hline & Menos del mínimo & 3 \\
\hline & No sabe & 4 \\
\hline
\end{tabular}

Notas: la valoración es de acuerdo con lo especificado por Rivera y Bernal (2018), pero la cantidad de preguntas está limitada a la información disponible en la ENIF.

Fuente: elaboración propia con información de la CNBV-INEGI-ENIF, 2018. Disponible en https://www.inegi.org.mx/programas/enif/2018/\#Datos_abiertos

\section{Tabla 3. Intervalos y frecuencias}

\begin{tabular}{cccccccc}
\hline \multicolumn{3}{c}{ Intervalos } & & \multicolumn{3}{c}{ Frecuencia } \\
\hline No & Lim. inf. & Lim. sup. & Marca clase & & Absoluta & Relativa & Acumulada \\
\hline Bajo & 0 & 2 & 1 & & 11906 & 95.661 & 11906 \\
Medio & 3 & 5 & 4 & & 529 & 4.250 & 12435 \\
Alto & 6 & 8 & 7 & & 11 & 0.088 & 12446 \\
Total & & & & 12446 & 100.000 & \\
\hline
\end{tabular}

Notas: la cantidad de preguntas está limitada a la información disponible en ENIF.

Fuente: elaboración propia con información de la CNBV-INEGIENIF, 2018. Disponible en https://www.inegi.org.mx/ programas/enif/2018/\#Datos_abiertos 
Con el fin de probar la hipótesis de que un individuo con mayor nivel en educación financiera puede aumentar sus niveles de ingreso, se utiliza el modelo propuesto por Villagómez y González (2014), donde:

$Y_{i}=$ Ingreso promedio mensual;

$E F_{i}=$ Índice de Educación Financiera;

$E_{i}=$ Nivel de educación;

$C_{i}=$ Variables de control para matizar las condiciones sociodemográficas de los individuos;

$U_{i}=$ Variables no observadas;

$\varepsilon_{i}=$ término de error.

La variable dependiente es el nivel de ingreso medio mensual, mientras que las independientes son el índice de educación financiera, nivel de educación y variables de control dicotómicas, que definen la región donde se ubica el individuo, el sexo, si es jefe(a) del hogar y la edad. Para el caso de la región se realizaron seis variables dicotómicas: la región 1 corresponde a los estados del noroeste: Baja California, Baja California Sur, Chihuahua, Durango y Sonora; la región 2 a la noreste: Coahuila, Nuevo León, San Luis Potosí y Tamaulipas; región 3 corresponden a estados del occidente-bajío: Aguascalientes, Guanajuato, Jalisco, Michoacán, Nayarit, Querétaro, Zacatecas y Colima; región 4 sólo considera a la Ciudad de México, debido a su magnitud y dinamismo particular; región 5 agrupa a los estados del centro del país: Estado de México, Hidalgo, Morelos, Puebla, Tlaxcala y Veracruz; finalmente, la región 6 corresponde al sur del país a los estados de Campeche, Chiapas, Guerrero, Quintana Roo, Tabasco, Yucatán y Oaxaca. La agrupación de los estados es aplicada por el INEGI en colaboración con la Comisión Nacional Bancaria y de Valores (CNBV), exclusivamente para la ENIF 2018.

\section{RESULTADOS Y DISCUSIÓN}

La medición de la educación financiera fue una labor que comenzó la oCDE en 2009, con el desarrollo de un método común que pudiera usarse para crear una medida de referencia, realizar seguimiento de los cambios a lo largo del tiempo, utilizarlo en países que integren el G20 y adaptarlo a las normas de cada país (OCDE, 2017). Con valores normalizados para 2017, el G20 tiene en promedio $60.47 \%$ en educación financiera. Entre los primeros lugares se encuentran Francia con $70.95 \%$, Noruega y Canadá con $69.52 \%$, mientras 
que México se ubica en el lugar 12 con $57.62 \%$. En contraste, las estimaciones realizadas en esta investigación, con datos de 2018, muestran que los mexicanos obtuvieron en promedio $58.63 \%$ en educación financiera (véase tabla 4), que corresponde a una diferencia de sólo 1\%, respecto al dato de la OCDE. Esto evidencia que, a pesar de los esfuerzos realizados desde el ámbito público, como la implementación de la ENEF, propuesta por la Secretaría de Hacienda y Crédito Público, sólo se ha generado un impacto marginal. Al desagregar el análisis por factores de interés, se observa inclusive una disminución del comportamiento y de las actitudes financieras, consistente con los resultados de Carpena et al. (2011) quienes revelan que mayor conocimiento financiero provoca cambios en las decisiones financieras, reflejando para el caso de México un aparente desincentivo a invertir en los mercados financieros.

Tabla 4. Índice de educación financiera

\begin{tabular}{lcc}
\hline & 2017 & 2018 \\
& OCDE & Propuesta \\
\hline Conocimiento & 19.52 & 22.72 \\
Comportamiento & 23.81 & 22.10 \\
Actitudes & 14.29 & 13.81 \\
\hline IEF & 57.62 & 58.63 \\
\hline
\end{tabular}

Notas: IEF: promedio ponderado de conocimiento, comportamiento y actitudes. Fuente: elaboración propia con información de la CNBV-INEGI-ENIF, 2018. Disponible en https://www.inegi.org.mx/programas/enif/2018/\#Datos_abiertos

Para el análisis de distribución del IEF, éste se estratificó por medio de STATA permitiendo, según la muestra, obtener tres categorías: bajo, medio y alto, en donde en el nivel bajo están aquellos individuos que tienen de 0 a $54.76 \%$, en nivel medio de 54.77 a $64.29 \%$ y en nivel alto de 64.30 a $100 \%$. Con base en la población representada a nivel nacional (véase tabla 5), en primer lugar, 38.94\% de la población cuenta con bajos niveles de educación financiera, lo que explica comportamientos y actitudes negativos. De acuerdo con Atkinson y Messy (2012), estos factores demuestran que los individuos no hacen valoraciones antes de realizar una compra, no administran sus gastos y sus ingresos, no planifican a futuro y no invierten en productos del mercado financiero. 
Tabla 5. Niveles de educación financiera

\begin{tabular}{lcc}
\hline & Frecuencia & Porcentaje \\
\hline Bajo & 4846 & 38.94 \\
Medio & 4079 & 32.77 \\
Alto & 3521 & 28.29 \\
Total & 12446 & 100.00 \\
\hline
\end{tabular}

Nota: los niveles de educación financiera fueron clasificados por medio de STATA. Fuente: elaboración propia con información de la CNBV-INEGI-ENIF, 2018. Disponible en https://www.inegi.org.mx/programas/enif/2018/\#Datos_ abiertos

En la figura 1 se observa que los individuos con altos niveles de ingreso tienen niveles de educación financiera también altos. Según Jappelli (2010), esto significa que individuos con altos niveles de educación financiera tienen mayor capacidad para generar oportunidades de inversión y mayor participación en los mercados financieros. De acuerdo con Ocampo (2014) estar financieramente educado permite a un individuo ser consciente de las decisiones que impactan diferentes etapas de su vida. Por el contrario, individuos con niveles de ingreso bajo, también se concentran en bajos niveles de educación financiera, lo que genera una ineficiente asignación de ahorro e inversión, que puede traducirse en la ineficiencia de los mecanismos del mercado financiero, provocando un círculo vicioso.

Respecto a los resultados del ejercicio econométrico se estimó, en la primera etapa, una regresión lineal por medio de mínimos cuadrados ordinarios (мСо). En principio, las pruebas estadísticas evidencian significancias individuales válidas, dado que los coeficientes son significativos al 95\% de confiabilidad, (véase tabla 7). De igual forma, se estimó una matriz de correlación de Pearson y una prueba de inflación de varianza (VIF), lo que descartó problemas de autocorrelación y colinealidad. La prueba de Breusch-Pagan revela presencia de heterocedasticidad, corregida por medio de una estimación lineal con errores estándar robustos. Como la variable dependiente se estimó en logaritmos, las magnitudes de los coeficientes se asocian a elasticidades a excepción de las dummys. 
Figura 1. Educación financiera e ingreso 2018

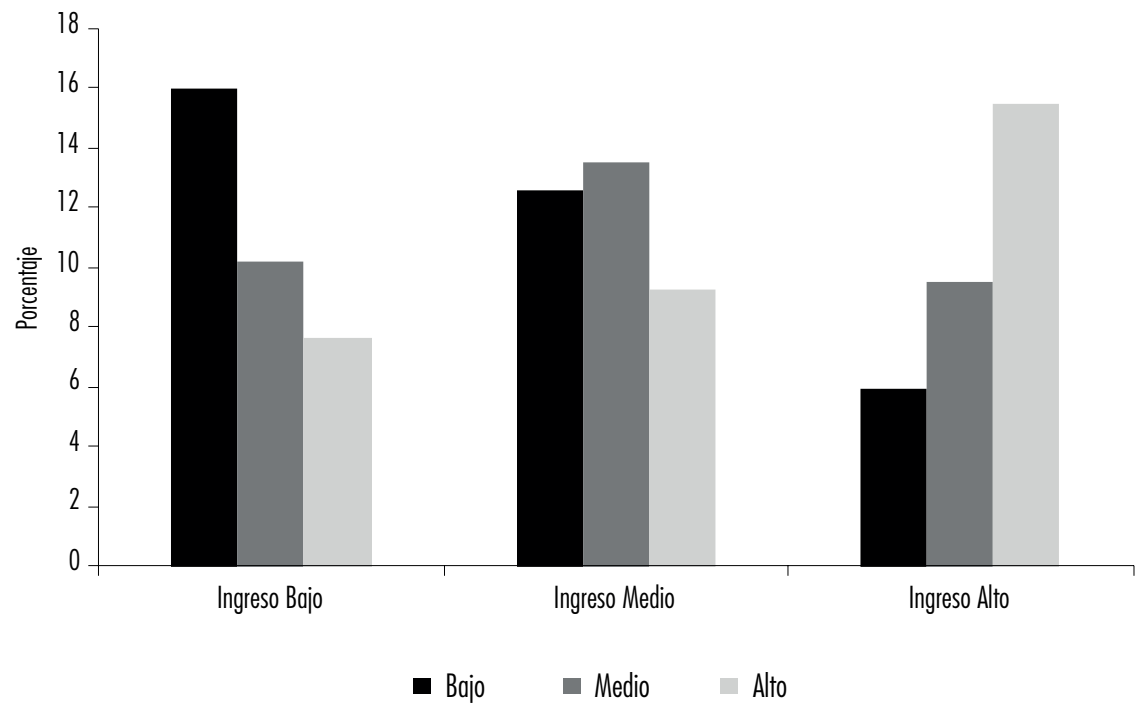

Fuente: elaboración propia con información de la CNBV-NEEGIENIF, 2018. Disponible en https://www.inegi.org.mx/programas/enif/2018/\#Datos_abiertos

Tabla 6. Estadística descriptiva para todas las variables

\begin{tabular}{lrrrrc}
\hline Estadísticos & Media & Desv Est & Max & Min & Observaciones \\
\hline Edad & 40.78 & 14.06 & 70 & 18 & 12446 \\
Género & 0.45 & 0.50 & 1 & 0 & 12446 \\
Región & 3.45 & 1.79 & 6 & 1 & 12446 \\
Jefe de Familia & 1.87 & 1.10 & 6 & 1 & 12446 \\
Dependientes & 1.58 & 1.62 & 25 & 0 & 12446 \\
Escolaridad & 4.47 & 3.36 & 8 & 0 & 12446 \\
Ocupación & 2.60 & 0.99 & 5 & 1 & 8991 \\
Ingreso Mensual & 6031.98 & 6544.26 & 98000 & 0 & 8570 \\
Seguro & 3.97 & 2.42 & 6 & 1 & 8570 \\
Índice de Educación Financiera & 58.63 & 11.79 & 90.48 & 0 & 12446 \\
\hline
\end{tabular}

Fuente: elaboración propia con información de la CNBV-NEGl-ENIF, 2018. Disponible en https://www.inegi.org.mx/ programas/enif/2018/\#Datos_abiertos 
Los resultados de la estimación indican que si una persona incrementa su nivel de educación financiera, en una unidad adicional en promedio, el ingreso mensual aumenta $1.75 \%$, lo que se interpreta como mayor inclusión social, pues se observa una relación directa entre ambas variables que permite ratificar la hipótesis inicial. Lo anterior coincide con el incremento en el conocimiento financiero de la población, y permite asociarlo con mejor equidad económica (Oliver et al., 2017). Para comprobar la causalidad del IEF y el ingreso, se realizó una prueba de mínimos cuadrados en segunda etapa (MC2E). Lo que permitió corregir problemas de endogeneidad y observar rendimientos de las variables. La prueba de causalidad sincrónica realizada para comprobar el rendimiento de IEF e ingreso, es consistente con la literatura, aun cuando es difícil comparar la causación entre ellas (García-Ferrer, 2008).

Por otra parte, los resultados de la estimación confirman la relación positiva entre la educación e ingreso, lo que se puede interpretar como que, ante el aumento de un año en escolaridad, el ingreso puede aumentar en $9.14 \%$, tema ampliamente discutido en trabajos de investigación aplicada y evidencia empírica (Lucas, 1988; García-Suaza et al., 2009; Lusardi y Mitchell, 2014; Lusardi et al., 2017).

Por su parte, el incremento en la edad presenta una relación directa con el ingreso, de tal forma que el ingreso aumenta $4.13 \%$ por año adicional de edad. Esto puede representar el impacto de una mayor experiencia, tanto laboral como en administración de recursos. Sin embargo, al analizar esta variable elevada exponencialmente (al cuadrado) se aprecia una relación negativa con el ingreso en $-0.05 \%$, lo que significaría que, a largo plazo, la edad tiene rendimientos marginales decrecientes, asociados a menores niveles de productividad laboral y menor acceso a servicios financieros, resultados que concuerdan con los encontrados en García-Suaza et al. (2009).

La variable de sexo muestra una vez más la inequidad existente entre hombres y mujeres en temas económicos. Ya que por el hecho de ser hombre se pueden obtener $42.96 \%$ más ingresos que las mujeres. Esta brecha se ha estudiado en Lusardi y Mitchell (2014), encontrando que factores como la educación, el tipo de puestos de trabajo, la generación de redes laborales, la disponibilidad de horarios, la discriminación laboral, son algunas de las causas que explican este sesgo (Federici, 2018; Larraz et al., 2019).

Respecto a la regionalización del país, es posible observar que el IEF varía con respecto a cada región y se confirma la relación positiva que tiene con el ingreso, según lo evidenciado por Jappelli (2010). Por ejemplo, en la región del noroeste $20.6 \%$ de la población se concentra en nivel alto de educación financiera, lo que corresponde a sus aportes en el Producto Interno Bruto 
Tabla 7. Estimaciones por MCO y MC2E

\begin{tabular}{|c|c|c|c|}
\hline Variables & Ingreso promedio mensual & Variables & Ingreso promedio mensual \\
\hline \multirow[t]{2}{*}{ IEF } & $0.0175408^{\star}$ & IEF & $0.0763577^{\star}$ \\
\hline & $(0.0007505)$ & & $(0.0024968)$ \\
\hline \multirow[t]{2}{*}{ Escolaridad } & $0.0914975^{\star}$ & & \\
\hline & $(0.0031302)$ & & \\
\hline \multicolumn{2}{|l|}{ Variables de Control } & Variables de Control & \\
\hline \multirow[t]{2}{*}{ Región 1} & $0.3543661^{*}$ & Región 1 & $0.3204813^{*}$ \\
\hline & $(0.0245284)$ & & (0.0328979) \\
\hline \multirow[t]{2}{*}{ Región 2} & $0.3011553^{\star}$ & Región 2 & $0.3304256^{*}$ \\
\hline & $(0.0247569)$ & & $(0.0330809)$ \\
\hline \multirow[t]{2}{*}{ Región 3} & $0.2835694^{\star}$ & Región 3 & $0.2797378^{\star}$ \\
\hline & (0.0249123) & & (0.0333168) \\
\hline \multirow[t]{2}{*}{ Región 4} & $0.2909745^{\star}$ & Región 4 & $0.2696577^{\star}$ \\
\hline & $(0.0349337)$ & & $(0.0468281)$ \\
\hline \multirow[t]{2}{*}{ Región 5} & $0.0719485^{\star}$ & Región 5 & $0.1217252^{\star}$ \\
\hline & $(0.0246295)$ & & $(0.032995)$ \\
\hline \multirow[t]{2}{*}{ Edad } & $0.0413054^{\star}$ & Edad & $0.0207331^{*}$ \\
\hline & (0.0037858) & & $(0.0051413)$ \\
\hline \multirow[t]{2}{*}{$\operatorname{Edad}^{\wedge} 2$} & $-0.0004885^{\star}$ & Edad $^{\wedge} 2$ & -0.0001879 \\
\hline & $(0.0000447)$ & & $(0.0000617)$ \\
\hline \multicolumn{2}{|l|}{ Variables № Observadas } & Variables No Observadas & \\
\hline \multirow[t]{2}{*}{ Sexo } & $0.4296106^{\star}$ & Sexo & $0.3925721^{*}$ \\
\hline & $(0.0168819)$ & & $(0.0225781)$ \\
\hline \multirow[t]{2}{*}{ Jefe de Familia } & $0.1831367^{\star}$ & Jefe de Familia & $0.1911067^{\star}$ \\
\hline & $(0.0179372)$ & & $(0.0240068)$ \\
\hline \multirow[t]{2}{*}{ Constante } & $5.658871^{\star}$ & Constante & $3.238916^{\star}$ \\
\hline & (0.0855348) & & (0.1723905) \\
\hline Observaciones & 7774 & Observaciones & 7774 \\
\hline Prob $>F$ & 0.0000 & Prob $>F$ & 0.0000 \\
\hline R-Cuadrada & 0.3444 & R-Cuadrada & - \\
\hline
\end{tabular}

Notas: errores estándar en paréntesis; ${ }^{*} \mathrm{p}<0.05$.

Fuente: elaboración propia. 
(Рів) nacional de $2.33 \%$, sobre todo las entidades de Baja California, Sonora y Chihuahua (INEGI, 2018). Es posible que las actividades de empresas industriales y de exportación en estas entidades estimulen a su capital humano a adquirir mayores conocimientos financieros, ya que los los derechos que se obtienen en las empresas permiten mayores facilidades para obtener servicios financieros (Mejía, 2016). Por otro lado, se observa que $9.91 \%$ se concentra en niveles altos en la Ciudad de México, lo que es posible debido a los grados de concentración de las instituciones financieras donde son más competitivas (BANXICO, 2013), se promueve el uso generalizado de productos financieros para su población y se generan mayores niveles de productividad. En el resto de las regiones no se aprecian diferencias significativas en los coeficientes, pero es relevante mencionar que el bajo nivel de educación financiera gira en relación directa a sus bajos niveles de desarrollo y la creciente participación de organizaciones de microcrédito, que fomentan el ahorro en esas regiones (Aguilar-Pinto et al., 2017).

De todo lo anterior, es posible señalar que hubo un crecimiento de la educación financiera entre la población, que generó confianza en la economía de mercado al permitir obtener asignaciones más eficientes de ahorros, inversión y bienestar. Lo anterior permite desarrollar habilidades y conciencia, tomar decisiones informadas y eficaces para mejorar el bienestar económico (Cartagena, 2008). De acuerdo con Lo Prete (2013), un crecimiento en educación financiera disminuye el crecimiento de la desigualdad como ocurre en países donde la educación económica es más alta entre la población.

Los niveles de endeudamiento son bajos (véase tabla 3), ya que $95.66 \%$ de los encuestados se ubican en el intervalo 0 a 2 . Lo que se corresponde con la información de la tabla 8 , donde $28.76 \%$ cuentan con algún crédito bancario, tarjeta de crédito bancaria, departamental, de tienda de autoservicio o de alguna otra institución financiera. Quienes tienen acceso a créditos, lo utilizan para aumentar su bienestar económico (Cartagena, 2008) mediante compras o mejoras del hogar, adquisición de terrenos o vehículos (23.6\%); alimentos, uso personal y servicios (18.3\%); y ampliar u operar un negocio para mejorar su calidad de vida (17.3\%). Sólo en este último caso el destino del crédito podrá tener efectos a la productividad, pues en los otros, es claro que los conocimientos financieros de los individuos son utilizados para tener menores niveles de endeudamiento para consumo a corto plazo.

Lo anterior permite afirmar que el destino de los créditos está ejerciendo una dinámica positiva, pues los individuos adquieren mayores conocimientos que les permiten comprender que sus niveles de endeudamiento se relacionan con sus capacidades económicas, lo que es consistente con lo evidenciado por 
Tabla 8. Crédito formal y destino

\begin{tabular}{|c|c|c|c|}
\hline Preguntas & Opciones de respuesta & Frecuencia & Porcentaje \\
\hline \multirow{2}{*}{$\begin{array}{l}\text { ¿Usted tiene algún crédito bancario, } \\
\text { tarijeta de crédito bancaria, } \\
\text { departamental, de tienda de } \\
\text { autoservicio o con alguna otra } \\
\text { institución financiera? }\end{array}$} & Si & 3580 & 28.76 \\
\hline & No & 8866 & 71.24 \\
\hline \multirow[t]{9}{*}{$\begin{array}{l}\text { ¿En qué utilizó o piensa utilizar } \\
\text { su(s) crédito(s)? }\end{array}$} & $\begin{array}{l}\text { Comprar, reparar, remodelar o ampliar una casa; } \\
\text { comprar terrenos, vehículos, joyas, animales, etcétera. }\end{array}$ & 317 & 28.64 \\
\hline & Gastos de comida, personales o pago de servicios. & 203 & 18.34 \\
\hline & $\begin{array}{l}\text { Comenzar, ampliar u operar un negocio (materia prima, } \\
\text { maquinaria y equipo). }\end{array}$ & 191 & 17.25 \\
\hline & Atender emergencias 0 imprevistos. & 82 & 7.41 \\
\hline & Pagar una deuda. & 97 & 8.76 \\
\hline & Gastos de salud. & 82 & 7.41 \\
\hline & Gastos de educación. & 72 & 6.50 \\
\hline & Pagar vacaciones o fiestas (XV años, bodas, etcétera). & 48 & 4.34 \\
\hline & Otro & 15 & 1.36 \\
\hline
\end{tabular}

Fuente: elaboración propia con información de la CNBV-INEGI-ENIF, 2018. Disponible en https://www.inegi.org.mx/programas/enif/2018/\#Datos_abiertos

Lusardi (2012). Sin embargo, persiste el problema del escaso porcentaje de mexicanos que cuentan con créditos formales de las instituciones financieras. De acuerdo con la tabla 9, 80.9\% de los encuestados no tienen problemas para cubrir el pago de sus tarjetas de crédito; $63.1 \%$ paga el monto total requerido para no generar intereses; y $97.7 \%$ no deja de pagar la mensualidad de su deuda con puntualidad, lo que indica comportamientos adecuados hacia el endeudamiento, lo que tiene sentido con lo argumentado por la CNBV (2012) respecto a que educación financiera permite el incremento de la inclusión financiera y del bienestar (Raccanello y Herrera, 2014).

La escolaridad mantiene una relación positiva con el tipo de ingreso de cada individuo, pues quienes cuentan con mayores grados de escolaridad (licenciatura o ingeniería) tendrán mayores niveles de educación financiera e incentivos para el uso racional de los productos del sistema financiero, así como mayor propensión a ser ciudadanos capaces de comprender y sacar provecho de políticas económicas y sociales. 
Tabla 9. Nivel de endeudamiento

\begin{tabular}{|c|c|c|c|}
\hline Preguntas & Opciones de respuesta & Frecuencia & Porcentaje \\
\hline \multirow[t]{3}{*}{ ¿Cuántas tarietas de crédito posee? } & 1 & 2498 & 60.76 \\
\hline & 2 & 866 & 21.07 \\
\hline & Más de 2 & 747 & 18.17 \\
\hline \multirow{3}{*}{$\begin{array}{l}\text { Durante el 2017, ¿ise ha atrasado en } \\
\text { alguna mensualidad? }\end{array}$} & Nunca & 2911 & 97.68 \\
\hline & Una vez & 13 & 0.44 \\
\hline & Más de una vez & 56 & 1.88 \\
\hline \multirow{2}{*}{$\begin{array}{l}\text { ¿Posee algún tipo de crédito o deuda } \\
\text { financiera distinto a la tarjeta de crédito? }\end{array}$} & Sí & 537 & 6.06 \\
\hline & № & 8329 & 93.94 \\
\hline \multirow{5}{*}{$\begin{array}{l}\text { ¿El pago que realiza a su tarjeta } \\
\text { generalmente cubre? }\end{array}$} & Requerido & 1965 & 63.10 \\
\hline & Más del pago mínimo & 554 & 17.79 \\
\hline & Pago mínimo & 448 & 14.39 \\
\hline & Menos del mínimo & 79 & 2.54 \\
\hline & No sabe & 68 & 2.18 \\
\hline
\end{tabular}

Fuente: elaboración propia con información de la CNBV-NEEG-ENIF, 2018. Disponible en hittps://www.inegi.org.mx/programas/enif/2018/\#Datos_abiertos

De los mexicanos encuestados, $54.69 \%$ son mujeres que se ubican entre niveles bajo-medio de educación financiera y se ocupan como empleadas o trabajadoras independientes (véase figura 2), muy posiblemente porque existe discriminación en el mismo sistema financiero, por temas de informalidad o por no contar con comprobantes de ingresos (Arcos-Medina et al., 2016). También Domínguez-Gijón et al. (2018) consideran que las mujeres tienen la capacidad de disminuir su consumo actual y mantenerlo constante durante el tiempo, para hacer frente a la incertidumbre futura. Por otro lado, $70.3 \%$ de los hombres con alto niveles de educación financiera cuenta con salarios altos, como empleados u obreros, muy probablemente porque al iniciar sus relaciones laborales adquieren beneficios como seguridad social y cuenta de nómina. Ello obliga a los empleados a aumentar sus niveles de educación financiera para el uso de cajeros automáticos y el uso correcto de su cuenta bancaria y de nómina, obteniendo servicios financieros por parte de las instituciones. De esta manera, los hombres representan $2.1 \%$ de la muestra, considerados emprendedores por tener alguna empresa y/o contratar empleados, tienden a tener altos niveles de educación financiera, lo que les permite ser capaces de 
Figura 2. Género, ocupación y nivel de educación financiera 2018

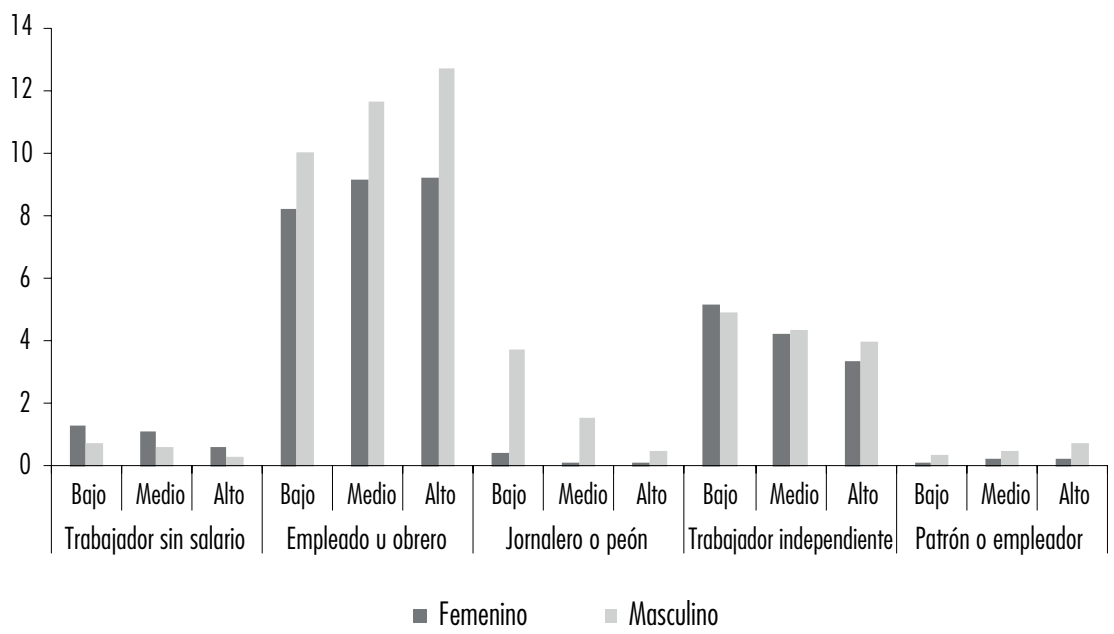

Fuente: elaboración propia con información de la CNBV-INEGI-ENIF, 2018. Disponible en https://www.inegi.org.mx/programas/enif/2018/\#Datos_abiertos

establecer objetivos a largo plazo y tomar decisiones efectivas para lograrlos; entender mejor el mercado financiero; y protegerse de cualquier práctica engańosa, por medio del aprendizaje (Martínez, 2017; Lusardi et al., 2017).

\section{CONCLUSIONES}

La estrategia de incrementar los niveles de educación financiera en México garantiza cambios en conocimientos, actitudes y comportamientos financieros. Aumentar los niveles de educación financiera puede mejorar a su vez los niveles de inclusión social y bienestar, sin importar el estrato social de los individuos, pues les permitiría adquirir servicios financieros en función su tipo y nivel de ingresos. Mayor educación financiera puede ocurrir ante una mayor reacción de la población a la creciente oferta de productos y servicios financieros, bajo esquemas de regulación, siempre y cuando se garantice la protección de los intereses de los consumidores, pues la adquisición de productos financieros dependerá de comportamientos y actitudes financieras positivas.

El esfuerzo público por aumentar la inclusión financiera, no se verá reflejado por sí mismo en aumentos racionales de educación financiera de las personas. Para que ello ocurra, el Estado tiene que proveer mecanismos y recursos para que el consumidor tenga la certeza de acercarse a instituciones y 
obtener la información y capacitación necesaria para utilizarlos correctamente. Esto permitiría obtener mayores beneficios y oportunidades de inversión a individuos con ingresos bajos mientras aumenten sus niveles de educación financiera.

Aunque México cuente con un sistema financiero con múltiples innovaciones, una importante parte de su población no cuenta con capacidades para aprovecharlo. Para que la educación financiera permita lograr mejores condiciones de vida debe ser integral e ir acompañada de un nivel de inclusión financiera, que permita una estrecha relación entre consumidor y servicios, y que aumente los niveles de bienestar. Una futura investigación asociada con la educación financiera puede desagregar la información a nivel regional en relación con el género y la cultura financiera. También puede analizar la evolución del conocimiento, comportamiento y actitudes financieras a lo largo del tiempo.

\section{BIBLIOGRAFÍA}

Aguilar-Pinto, E. D. C., Tuñón-Pablos, E. y Morales-Barragán, F. (2017). Microcrédito y pobreza. La experiencia del programa microempresas sociales de Banmujer en Chiapas. Economia, Sociedad y Territorio, 17(55). https:// doi.org/http://dx.doi.org/10.22136/est002017635

Aparicio, A. (2010). Economía mexicana 1910-2010: balance de un siglo. ECOES Facultad de Economía UNAM. www.economia.unam.mx/profesores/aaparicio/Economía.pdf

Araoz-Fraser, S. (2010). Inclusión social: un propósito nacional para Colombia. En F. U. Central (1a. ed.). Documentos de Investigación-Economia (pp. 1-50). Universidad Central. https://www.ucentral.edu.co/sites/default/ files/inline-files/2010-12-documentos-investigacion-economia-007.pdf

Arcos-Medina, G., Hernández-Romero, O. y Zapata-Martelo, E. (2016). Ahorro infantil, un acercamiento a la inclusión financiera. Chispitas de la Fundación Ayú, Oaxaca, México. Agricultura, Sociedad y Desarrollo, 13(3). http://www.redalyc.org/pdf/3605/360547924008.pdf

Atkinson, A. y Messy, F. (2012). Measuring financial literacy: Results of the OECD/International Network on Financial Education (INFE) Pilot Study. OECD Working Papers on Finance, Insurance and Private Pensions, 15. http://dx.doi.org/10.1787/5k9csfs90fr4-en\%0AOECD

Banco Mundial (2014). Inclusión social: clave de la prosperidad para todos. https:/openknowledge.worldbank.org/bitstream/handle/10986/16195/ 817480WP0Spani0IC00InclusionMatters.pdf? sequence $=6 \&$ isAllowed $=y$ 
BANXICO (2013). Reporte sobre las condiciones de competencia en el mercado de emisión de tarjetas de crédito. https://www.banxico.org.mx/publi caciones-y-prensa/reportes-sobre-las-condiciones-de-competencia-en1/\%7B9A9ADEB4-7D4E-8307-B645-DB78A8A91ADE\%7D.pdf?fbclid $=$ IwAR0YgXCTKenRH8DyI4Cmw_CymMytRxJWeDL2F2teQvnNNn 0pIS3VILIn_4c

(2018). Crecimiento económico y productividad. https://www.ban xico.org.mx/publicaciones-y-prensa/informes-trimestrales/recuadros/\% 7B81E83E11-CFE1-C761-B850-8949FC923C55\%7D.pdf

(2019). Indicadores básicos de tarjetas de crédito. https:// www.banxico.org.mx/publicaciones-y-prensa/rib-tarjetas-decredito/\%7B1F51BE6A-0A37-6043-8FEB-0B57D9CDC0E8\%7D.pdf

Carpena, F., Cole, S., Shapiro, J. y Zia, B. (2011). Unpacking the causal chain of financial literacy. Policy Research Working Paper, 5798. https://doi. org/10.1596/1813-9450-5798

Cartagena, E. (2008). La educación financiera, como un pilar para el desarrollo financiero de los países: una aplicación para El Salvador. Tópicos Económicos, 1(24). https://www.bcr.gob.sv/bcrsite/downloads.php?dta=397

Ceballos, O. E. (2018). Perfiles de ahorro y pago de deuda en el ciclo de vida de los hogares mexicanos. El Trimestre Económico, 85(338). Recuperado de https://www.readcube.com/articles/10.20430/ete.v85i338.322

Céspedes, J. B. (2017). Análisis de la necesidad de la educación financiera en la formación colegial. Pensamiento Crítico, 22(2). https://doi.org/10.15381/ pc.v22i2.14333

Comisión Nacional Bancaria y de Valores (CNBv) (2012). Libro blanco inclusión financiera. http://www.google.com.mx/\#hl=es\&sclient=psy-ab\&q= Libro+blanco+inclusión+financiera+pdf\&oq=Libro+blanco+inclusión + financiera+pdf\&gs_l=hp.3...5518.6819.2.7858.4.4.0.0.0.0.152.569. 0j4.4.0...0.0...1c.1.12.psy-ab.DrpiKIZ0ZsU\&pbx=1\&bav=on.2,or.r_ qf. \&bvm=bv. 4634

Comisión Nacional Bancaria y de Valores-Encuesta Nacional de Inclusión Financiera (CNBV-ENIF) (2015). Encuesta Nacional de Inclusión Financiera, 261. https://www.cnbv.gob.mx/Inclusión/Documents/Encuesta Nacional de IF/ENIF 2015.pdf

Comisión Nacional Bancaria y de Valores-Instituto Nacional de Estadística y Geografía-Encuesta Nacional de Inclusión Financiera (CNBV-INEGI-ENIF) (2018). Encuesta Nacional de Inclusión Financiera. https://www.inegi. org.mx/programas/enif/2018/\#Datos_abiertos 
Córdova-Rangel, A., Zamora-Lobato, T., Moreno-García, E. y GarcíaSantillán, A. (2016). El nivel de educación financiera de los jóvenes de bachillerato y su influencia en la percepción que tienen de las instituciones financieras. Un estudio empirico. February 2017. https://www.researchgate.net/ publication/313608875\%0AEL

De la Cruz, J. L. y Alcántara, J. Á. (2011). Crecimiento económico y el crédito bancario: un análisis de causalidad para México. Revista de Economia, 28(77). https://doi.org/10.33937/reveco.2011.25

De la Cruz, J. L. y Veintimilla, D. V. (2013). Banca de desarrollo y crecimiento económico en México, la necesidad de una nueva estrategia. Análisis, 5. http://library.fes.de/pdf-files/bueros/mexiko/10214.pdf

Denegri, M., Sepúlveda, J. y Silva, F. (2018). Comprender la economía: educación económica y financiera en la infancia desde una perspectiva constructivista. Ensino Em Revista, 25(1). http://www.seer.ufu.br/index.php/ emrevista/article/download/41360/21867/

Diez, E. (2009). La alfabetización socioeconómica y financiera y la educación para el consumo sostenible en México: algunas reflexiones desde la psicología y la educación. Revista de Investigación Educativa, 8. https://www. redalyc.org/pdf/2831/283121717005.pdf

Domínguez-Gijón, R. M., Venegas-Martínez, F. y García-Ruíz, R. S. (2018). Un modelo microeconómico estocástico del comportamiento de una jefa de familia como único participante en el ingreso familiar: el caso mexicano, 2005-2016. Economía Teoría y Práctica, 49. https://doi.org/10.24275/ ETYPUAM/NE/492018/Dominguez

Duque, E., González, J. D. y Ramírez, J. D. (2016). Conocimientos financieros en jóvenes universitarios: caracterización en la institución universitaria ESUMER. Revista de Pedagogia, 37(101). https://www.researchgate.net/publication/315676004

Federici, S. (2018). El patriarcado del salario: criticas feministas al marxismo. https://www.traficantes.net/sites/default/files/pdfs/TDS_map49_federici_web_0.pdf

García, N., Grifoni, A., López, J. C. y Mejía, D. M. (2013). La educación financiera en América Latina y el Caribe. Situación actual y perspectivas. Serie Politicas Públicas y Transformación Productiva, 12. https://doi. org/10.1029/JD095iD05p05697

García-Ferrer, A. (2008). Causalidad y econometría. En J. C. García Bermejo (Ed.), Sobre la economía y sus métodos (pp. 225-240). Trotta, Csic. https://www.researchgate.net/publication/233756636_Causalidad_y_ Econometria 
García-Suaza, A. F., Guataquí, J. C., Guerra, J. A. y Maldonado, D. (2009). Beyond the mincer equation: the internal rate of return to higher education in Colombia. Universidad Del Rosario, 68. https://doi.org/10.1080/0 9645292.2011 .595579

Huchín, L. A. y Simón, J. D. (2011). La educación financiera estudiantes de educación básica. Un diagnóstico comparativo entre escuelas urbanas y rurales. Revista Ciencias Estratégicas, 19(25). https://revistas.upb.edu.co/ index.php/cienciasestrategicas/article/view/1101

Instituto Nacional de Estadística y Geografía (INEGI) (2018). Producto Interno Bruto por Entidad Federativa. https://www.inegi.org.mx/contenidos/ saladeprensa/boletines/2019/OtrTemEcon/PIBEntFed2018.pdf

Jappelli, T. (2010). Economic literacy: An international comparison. Economic Journal, 120(548). https://doi.org/10.1111/j.1468-0297.2010.02397.x

Larraz, B., Pavia, J. M. y Vila, L. E. (2019). Beyond the gender pay gap. Más allá de la brecha salarial de género. Convergencia Revista de Ciencias Sociales, 81. https://doi.org/10.29101/crcs.v26i81.11579

Lo Prete, A. (2013). Economic literacy, inequality, and financial development. Economics Letters, 118(1). https://doi.org/10.1016/j.econlet.2012.09.029 (2017). Inequality and the finance you know: Does economic literacy matter? The Department of Economics and Statistics "Cognetti de Martiis". https://www.researchgate.net/publication/322799255\%0A

Lucas, R. E. (1988). On the mechanics of economic development. Monetary Economics, 22. https://www.parisschoolofeconomics.eu/docs/darcillon-thi bault/lucasmechanicseconomicgrowth.pdf

Lusardi, A. (2008). Financial literacy: An essential tool for informed consumer choice? NBER Working Paper, 14084. http://www.nber.org/papers/ w1 14084

(2012). Numeracy, financial literacy, and financial decision-making. National Bureau of Economic Research, 17821. https:/doi.org/10.5038/ 1936-4660.5.1.2

Lusardi, A. y Mitchell, O. S. (2014). The economic importance of financial literacy: Theory and evidence. Journal of Economic Literature, 52(1). https://www.nber.org/papers/w18952

, Michaud, P.-C. y Mitchell, O. S. (2017). Optimal financial knowledge and wealth inequality. Journal of Political Economy, 152(2). https://doi.org/10.2139/ssrn.2585222

Martínez, I. I. (2017). Cinco principios para una educación financiera efectiva y cómo utilizarlos para la educación al consumidor. Boletín APCFC. https://www.researchgate.net/publication/324278958 
Mejía, G. (2016). Impacto de las capacidades financieras en el bienestar de los empleados. Revista de Estudios en Contaduría, Administración e Informática, 11. https://www.researchgate.net/publication/301489156

Ocampo, M. (2014). Sistema bancario colombiano y la educación financiera. Caso banco Davivienda. Aglala, 5(1). https://doi.org/https://doi. org/10.22519/22157360.757

OCDE (2017). G20/OECD INFE report on adult financial literacy in G20 countries. https://www.oecd.org/daf/fin/financial-education/OECD-INFE-In ternational-Survey-of-Adult-Financial-Literacy-Competencies.pdf

OCDE-CVM (2005). Recomendación sobre los principios y buenas prácticas de educación y concienciación financiera. www.oecd.org/finance/financial-edu cation/35108560.pdf

Oliver, F. J., Amate, I. y Guarnido, A. (2017). El conocimiento financiero y su impacto en la desigualdad de la renta. XXIV Encuentro de Economía Pública. https://dialnet.unirioja.es/descarga/articulo/6121555.pdf

Raccanello, K. y Herrera, E. (2014). Educación e inclusión financiera. Revista Latinoamericana de Estudios Educativos (México), 44(2). http://search. ebscohost.com/login.aspx?direct=true \&db=ofm\&AN=97317726\&lang= es\&site=ehost-live

Rivera, B. E. y Bernal, D. (2018). La importancia de la educación financiera en la toma de decisiones de endeudamiento. Estudio de una sucursal de "Mi Banco" en México. Perspectivas, 41. http://www.scielo.org.bo/pdf/rp/ n41/n41_a06.pdf

Sánchez, I. L. y Moreno, J. C. (2016). El reto del crecimiento en México: industrias manufactureras y política industrial. Revista Finanzas y Politica Económica, 8(2). https://doi.org/10.14718/revfinanzpolitecon.2016.8.2.4

Sauza, B., Pérez, S. S., Cruz, D., Lechuga, C. B. y Ramírez, M. G. (2018). Educación financiera y su relación con el uso de la tarjeta de crédito. Ingenio y Conciencia Boletín Cientifico de la Escuela Superior. https://doi.org/ https://doi.org/10.29057/ess.v4i8.2352

Villagómez, A. e Hidalgo, J. A. (2017). Financial literacy and mathematics: A study among young Mexican High School students. Revista Mexicana de Economia y Finanzas, 12(2). https://www.redalyc.org/articulo. oa?id $=423750525001$

Villagómez, F. A. y González, A. (2014). El efecto del alfabetismo financiero en el ahorro para el retiro en México. Documentos de Trabajo CIDE, 576. https://ideas.repec.org/p/emc/wpaper/dte576.html

Widdowson, D. y Hailwood, K. (2007). Financial literacy and its role in promoting a sound financial system. Reserve Bank of New Zealand: Bulletin, 70(2). https://doi.org/10.4161/21565562.2014.983399 